\title{
Datenerhebung und Analyse
}

Die Zielsetzung der Arbeit besteht in der Identifikation, Strukturierung und Generalisierung von Gestaltungsaspekten der Digital Customer Experience in der Videospielindustrie. Die vorgestellten Ergebnisse basieren auf einer Analyse der Videospielplattform Steam. Datenerhebung und Analyse folgen der Einzelfallstudienmethodik nach Yin (2013). Im Rahmen der Datenerhebung wurde die Entwicklung von Steam zwischen 2003 und 2016, d. h. die Implementierung von Features und Services sowie die Entwicklung von Nutzerzahlen erfasst. Zudem wurden öffentlich zugängliche Unternehmensinformationen erhoben und Publikationen von Experten in der Spieleindustrie recherchiert. Schließlich wurde ergänzend der Steam-Client installiert und einzelne Features untersucht.

Die Analyse der Gestaltung digitaler Plattformen für die Kundeninteraktion folgt der sozio-technischen Systemtheorie, welche sowohl eine technische als auch eine soziale Perspektive auf Steam ermöglicht. In der sozio-technischen Systemtheorie werden neben den beteiligten Akteuren (z. B. Spieler, Entwickler und Valve) auch die bereitgestellten Services, die eingesetzten Technologien sowie die handlungsleitenden sozialen Strukturen, z. B. Werte und Normen, berücksichtigt (Bostrom und Heinen 1977). Aus der Synthese der zugrunde liegenden wissenschaftlichen Literatur ergeben sich drei Gestaltungsebenen und deren Kernfragen (Bostrom und Heinen 1977; Lyytinen und Newman 2008), die nachfolgend eingeführt und in Bezug auf die Digital Customer Experience vorgestellt werden. Auf Basis dieses theoretischen Rahmens werden anschließend die Ergebnisse der Analyse von Steam präsentiert. 